\title{
Influence of oil and gas field operations on spatial and temporal distributions of atmospheric non-methane hydrocarbons and their effect on ozone formation in winter
}

\author{
R. A. Field et al.
}

Correspondence to: R. A. Field (rfield1@uwyo.edu) 


\section{Supplemental Materials}

Table S1 Measurement methodologies

\begin{tabular}{|c|c|c|c|c|c|c|}
\hline Parameter $^{1}$ & Method \& Instrument model & Averaging Time & Linearity & Precision & Accuracy & $\mathrm{LDL}^{3}$ \\
\hline $\mathrm{O}_{3}$ & $\begin{array}{l}\text { UV Photometric (Thermo } \\
55 \mathrm{i})\end{array}$ & $5-\min$ & $\pm 1 \%$ full scale & $\pm 1.0 \mathrm{ppbv}$ & $< \pm 5 \%$ & $0.4 \mathrm{ppbv}$ \\
\hline $\mathrm{CO}$ & NDIR (Thermo 48i TLE) & $1 \mathrm{~min}$ & $\pm 1 \%$ full scale & $\pm 10 \mathrm{ppbv}$ & $< \pm 10 \%$ & $0.04 \mathrm{ppmv}$ \\
\hline $\begin{array}{l}\mathrm{NO}-\mathrm{NO}_{2-} \\
\mathrm{NO}_{\mathrm{x}}\end{array}$ & $\begin{array}{l}\text { Chemiluminescence } \\
\text { (Thermo 42i) }\end{array}$ & 5-min & $\pm 1 \%$ full scale & $\pm 0.4 \mathrm{ppbv}$ & $< \pm 5 \%$ & $0.4 \mathrm{ppbv}$ \\
\hline $\begin{array}{l}\mathrm{CH}_{4} \text { and } \\
\mathrm{NMHC}\end{array}$ & $\begin{array}{l}\text { GC/FID } \\
\text { (Thermo 55i) }\end{array}$ & $1 \mathrm{~min}$ & $\begin{array}{l} \pm 1 \%(10 \% \text { to } \\
90 \% \text { range })\end{array}$ & $\begin{array}{l}<2 \% \text { or } \\
50 \mathrm{ppbv}\end{array}$ & $< \pm 5 \%$ & $10 \mathrm{ppbv}$ \\
\hline $\begin{array}{l}\text { Speciated } \\
\text { NMHC }^{2}\end{array}$ & $\begin{array}{l}\text { GC/FID (Perkin Elmer OPA } \\
\text { system) }\end{array}$ & $\begin{array}{l}\text { Grab, 1-hr, 24- } \\
\text { hour \& 3-day }\end{array}$ & $\begin{array}{l}< \pm 2 \% \\
\text { calibration range }\end{array}$ & $\begin{array}{l}<4 \% \text { or } \\
0.01 \mathrm{ppbv}\end{array}$ & $< \pm 3 \%$ & $0.01 \mathrm{ppbv}$ \\
\hline
\end{tabular}

${ }^{1}$ A Vaissla WXT520 was used for meteorological measurements of wind speed, wind direction, barometric pressure, relative humidity and temperature

${ }^{2}$ Sampling performed either on-line or through attachment of 6L Entech Inc. Silonite coated canisters that were filled from vacuum to ambient pressure using Entech Inc. CS1200E samplers

${ }^{3}$ LDL: Lower detection limit 
Table S2 Speciated NMHC canister monitoring site descriptions and sampling frequency

\begin{tabular}{|c|l|l|c|c|c|l|}
\hline No. & Site name & Co-ordinates & $2010-11$ & $2011-12$ & $2012-13$ & Category \\
\hline 1 & Dry Piney & $42.4115,-110.1206$ & 2 & & & Background NW \\
\hline 2 & Big Piney HS & $42.5391,-110.1207$ & 2 & & & Background NW \\
\hline 3 & WDEQ Juel Spring & $42.3716,-109.5504$ & 3 & & & Background S \\
\hline 4 & Speedway CD Trail Irish Canyon & $42.6437,-109.4704$ & 4 & & & Background E \\
\hline 5 & Calpet Road & $42.3810,-110.2837$ & 2 & & & Background W \\
\hline 6 & WDEQ Daniel & $42.7913,-110.0543$ & 2 & & & Background W \\
\hline 7 & WDEQ Pinedale & $42.7913,-110.0543$ & 2 & & & Background N \\
\hline 8 & Luman road & $42.4656,-109.5776$ & 4 & & & Background S \\
\hline 9 & Buckhorn & $42.3522,-109.8543$ & 4 & & & Background S \\
\hline 10 & WDEQ Boulder (BLDR) & $42.7184,-109.7522$ & 6 & 13 & 16 & Boundary Pinedale Anticline \\
\hline 11 & Olson Ranch & $42.5993,-109.8696$ & 3 & 12 & & Boundary Pinedale Anticline \\
\hline 12 & Boulder Lake & $42.7688,-109.6712$ & 2 & & & Background E \\
\hline 13 & Warbonnet & $42.5671,-109.7018$ & 4 & & & Pinedale Anticline \\
\hline 14 & Mesa North & $42.7530,-109.8577$ & 4 & 13 & 17 & Pinedale Anticline \\
\hline 15 & Mesa South & $42.7065,-109.8232$ & 4 & 11 & & Pinedale Anticline \\
\hline 16 & Yellowpoint Studhorse & $42.4508,-109.6899$ & 2 & & & Background S (Jonah Field) \\
\hline 17 & Mesa TOP & $42.8357,-109.8838$ & 2 & 13 & & Boundary Pinedale Anticline \\
\hline 18 & Middle Fork & $42.5430,-109.6614$ & 2 & 13 & & Boundary Pinedale Anticline \\
\hline 19 & Mesa Middle & $42.7396,-109.8427$ & 2 & & & Pinedale Anticline \\
\hline 20 & Paradise Road & $42.6806,-109.7956$ & 2 & 12 & & Pinedale Anticline \\
\hline 21 & Boulder 351 & $42.6032,-109.7285$ & 2 & 12 & & Pinedale Anticline \\
\hline 22 & Boulder Crest & $42.6593,-109.7845$ & 2 & 12 & 14 & Pinedale Anticline \\
\hline 23 & Middle Crest & $42.6274,-109.7540$ & 2 & & 3 & Pinedale Anticline \\
\hline 24 & UW Boulder South Road (BSR) & $42.6840,-109.7083$ & & 12 & & Boundary Pinedale Anticline \\
\hline 25 & Hennick Draw & $42.8032,-109.9623$ & & & 13 & Background NW \\
\hline 26 & Mesa South Loop & $42.7283,-109.8604$ & & & 4 & Pinedale Anticline \\
\hline 27 & Pinedale Complex & $42.6887,-109.8082$ & & & 2 & Pinedale Anticline \\
\hline 28 & Anticline Disposal Facility & $42.6490,-109.7617$ & & 23 & 9 & Pinedale Anticline \\
\hline
\end{tabular}


Table S3 Audit test results 2012

\begin{tabular}{|l|c|c|}
\hline Species & Reported (ppbv) & Audit Level (ppbv) \\
\hline ethane & 33.5 & 36.5 \\
\hline ethene (ethylene) & 18.6 & 18.0 \\
\hline propane & 30.3 & 33.1 \\
\hline propene (propylene) & 5.5 & 6.4 \\
\hline 2-methylpropane $(i$-butane) & 16.1 & 16.7 \\
\hline Butane $(n$-butane) & 30.6 & 30.5 \\
\hline trans-but-2-ene $(t$-2-butene) & 0.0 & \\
\hline 1-butene (but-1-ene) & 0.0 & \\
\hline cis-but-2-ene $(c-2$-butene) & 9.7 & 9.8 \\
\hline cyclopentane & 0.0 & \\
\hline 2-methylbutane $(i$-pentane) & 22.3 & 22.2 \\
\hline pentane $(i$-pentane) & 26.7 & 26.8 \\
\hline 1,3-butadiene & 0.0 & \\
\hline prop-1-yne (propyne) & 0.0 & \\
\hline trans-pent-2-ene $(t$-2-pentene) & 0.0 & \\
\hline 1-pentene & 0.0 & \\
\hline cis-pent-2-ene $(c-2$-pentene) & 10.5 & \\
\hline 2-methylpentane $(i$-hexane) & 0.0 & \\
\hline 3-methylpentane & 0.0 & \\
\hline hexane $(n$-hexane) & 11.9 & \\
\hline benzene & 11.7 & 10.7 \\
\hline cyclohexane & 0.0 & 11.6 \\
\hline Heptane $(n$-heptane) & 22.7 & \\
\hline toluene & 21.1 & \\
\hline octane & 3.7 & \\
\hline ethyl-benzene & 7.2 & \\
\hline$m+p$-Xylene & 15.9 & \\
\hline styrene & 0.0 & \\
\hline$o$-Xylene & 5.2 & \\
\hline
\end{tabular}


Table S4 Summary statistics of hourly measurements at Boulder South Road (BSR) 10/31/2010 to $3 / 18 / 2011$

\begin{tabular}{|c|c|c|c|c|c|c|c|c|c|}
\hline & Units & No. & Min. & $\begin{array}{l}25^{\text {th }} \\
\% \text { tile }\end{array}$ & $\begin{array}{l}50^{\text {th }} \\
\% \text { tile }\end{array}$ & Av. & $\begin{array}{l}75^{\text {th }} \\
\% \text { tile }\end{array}$ & $\begin{array}{l}95^{\text {th }} \\
\% \text { tile }\end{array}$ & Max. \\
\hline Wind Speed & $\mathrm{m} / \mathrm{s}$ & 3222 & 0.1 & 1.6 & 2.3 & 3.2 & 4.2 & 8.5 & 13.6 \\
\hline Temperature & ${ }^{\circ} \mathrm{C}$ & 3222 & -31.9 & -8.3 & -4.2 & -5.3 & -0.7 & 2.8 & 16.5 \\
\hline Relative Humidity & $\%$ & 3222 & 29 & 69 & 79 & 77 & 86 & 92 & 96 \\
\hline $\mathrm{O}_{3}$ & ppbv & 3303 & 0 & 30 & 38 & 38 & 44 & 59 & 119 \\
\hline $\mathrm{CO}$ & ppbv & 3303 & 90 & 139 & 156 & 167 & 180 & 250 & 850 \\
\hline $\mathrm{NO}$ & ppbv & 3303 & 0 & 0 & 0 & 1 & 1 & 4 & 19 \\
\hline $\mathrm{NO}_{2}$ & ppbv & 3303 & 0 & 2 & 4 & 5 & 6 & 13 & 42 \\
\hline $\mathrm{NO}_{\mathrm{x}}$ & ppbv & 3303 & 0 & 2 & 4 & 6 & 8 & 16 & 50 \\
\hline Methane & ppmv & 3209 & 1.80 & 1.93 & 2.06 & 2.22 & 2.29 & 3.27 & 6.71 \\
\hline NMHC & ppmC & 3209 & 0.00 & 0.05 & 0.12 & 0.23 & 0.26 & 0.77 & 7.40 \\
\hline ethane & ppbv & 2389 & 0.8 & 5.9 & 13.9 & 23.2 & 29.3 & 79.8 & 230.5 \\
\hline ethene & ppbv & 2389 & 0.0 & 0.2 & 0.5 & 0.7 & 0.9 & 2.4 & 8.5 \\
\hline propane & ppbv & 2389 & 0.2 & 2.5 & 5.7 & 8.9 & 11.5 & 28.6 & 82.6 \\
\hline propene & ppbv & 2389 & 0.0 & 0.1 & 0.1 & 0.2 & 0.2 & 0.4 & 1.2 \\
\hline$i$-butane & ppbv & 2389 & 0.0 & 0.5 & 1.3 & 2.1 & 2.8 & 7.0 & 21.3 \\
\hline$n$-butane & ppbv & 2389 & 0.1 & 0.7 & 1.5 & 2.3 & 3.1 & 7.4 & 22.5 \\
\hline ethyne & ppbv & 2392 & 0.0 & 0.3 & 0.5 & 1.0 & 1.1 & 3.5 & 15.5 \\
\hline$t$-2-butene & ppbv & 2389 & 0.0 & 0.1 & 0.1 & 0.2 & 0.2 & 0.3 & 0.5 \\
\hline 1-butene & ppbv & 2389 & 0.0 & 0.0 & 0.0 & 0.0 & 0.0 & 0.0 & 0.1 \\
\hline$c$-2-butene & $\mathrm{ppbv}$ & 2389 & 0.0 & 0.0 & 0.0 & 0.0 & 0.1 & 0.2 & 0.5 \\
\hline cyclopentane & ppbv & 2389 & 0.0 & 0.0 & 0.1 & 0.1 & 0.1 & 0.4 & 2.0 \\
\hline$i$-pentane & ppbv & 2389 & 0.0 & 0.3 & 0.8 & 1.3 & 1.6 & 4.1 & 14.1 \\
\hline$n$-pentane & ppbv & 2389 & 0.0 & 0.2 & 0.6 & 0.9 & 1.2 & 3.1 & 13.6 \\
\hline 1,3-butadiene & ppbv & 2389 & 0.0 & 0.0 & 0.0 & 0.0 & 0.0 & 0.0 & 0.1 \\
\hline propyne & ppbv & 2389 & 0.0 & 0.0 & 0.0 & 0.0 & 0.0 & 0.1 & 0.1 \\
\hline 1-pentene & ppbv & 2389 & 0.0 & 0.0 & 0.0 & 0.0 & 0.0 & 0.0 & 0.0 \\
\hline 2-methylpentane & ppbv & 2389 & 0.0 & 0.1 & 0.2 & 0.4 & 0.4 & 1.5 & 12.1 \\
\hline 3-methylpentane & ppbv & 2389 & 0.0 & 0.0 & 0.1 & 0.2 & 0.3 & 0.9 & 8.1 \\
\hline$n$-hexane & ppbv & 2389 & 0.0 & 0.1 & 0.3 & 0.6 & 0.7 & 2.3 & 20.1 \\
\hline isoprene & ppbv & 2389 & 0.0 & 0.0 & 0.0 & 0.0 & 0.0 & 0.0 & 0.0 \\
\hline benzene & ppbv & 2389 & 0.0 & 0.3 & 0.6 & 1.0 & 1.2 & 3.3 & 16.0 \\
\hline cyclohexane & ppbv & 2389 & 0.0 & 0.1 & 0.3 & 0.6 & 0.7 & 2.4 & 22.2 \\
\hline$i$-octane & ppbv & 2389 & 0.0 & 0.1 & 0.1 & 0.2 & 0.2 & 0.5 & 4.5 \\
\hline$n$-heptane & ppbv & 2389 & 0.0 & 0.1 & 0.3 & 0.6 & 0.6 & 2.2 & 19.7 \\
\hline toluene & ppbv & 2389 & 0.0 & 1.0 & 1.7 & 2.8 & 3.0 & 7.6 & 274.4 \\
\hline$n$-octane & ppbv & 2389 & 0.0 & 0.1 & 0.2 & 0.4 & 0.4 & 1.6 & 17.3 \\
\hline ethylbenzene & ppbv & 2389 & 0.0 & 0.1 & 0.2 & 0.2 & 0.2 & 0.5 & 4.3 \\
\hline$m+p$-xylene & ppbv & 2389 & 0.0 & 0.4 & 0.7 & 1.2 & 1.3 & 3.7 & 37.9 \\
\hline styrene & ppbv & 2389 & 0.0 & 0.1 & 0.2 & 0.2 & 0.2 & 0.4 & 1.8 \\
\hline$o$-xylene & ppbv & 2389 & 0.0 & 0.1 & 0.2 & 0.3 & 0.3 & 0.7 & 7.1 \\
\hline nonane & ppbv & 2389 & 0.0 & 0.1 & 0.1 & 0.4 & 0.3 & 1.2 & 14.4 \\
\hline Factor 1 & $*$ & 2311 & -0.20 & 0.60 & 0.85 & 1.00 & 1.22 & 2.27 & 4.24 \\
\hline Factor 2 & $*$ & 2311 & -0.20 & 0.12 & 0.52 & 1.00 & 1.30 & 3.86 & 14.05 \\
\hline Factor 3 & $*$ & 2311 & -0.20 & 0.21 & 0.42 & 1.00 & 0.96 & 3.56 & 41.97 \\
\hline
\end{tabular}

*Factor contribution of each hour relative to an average value of 1 


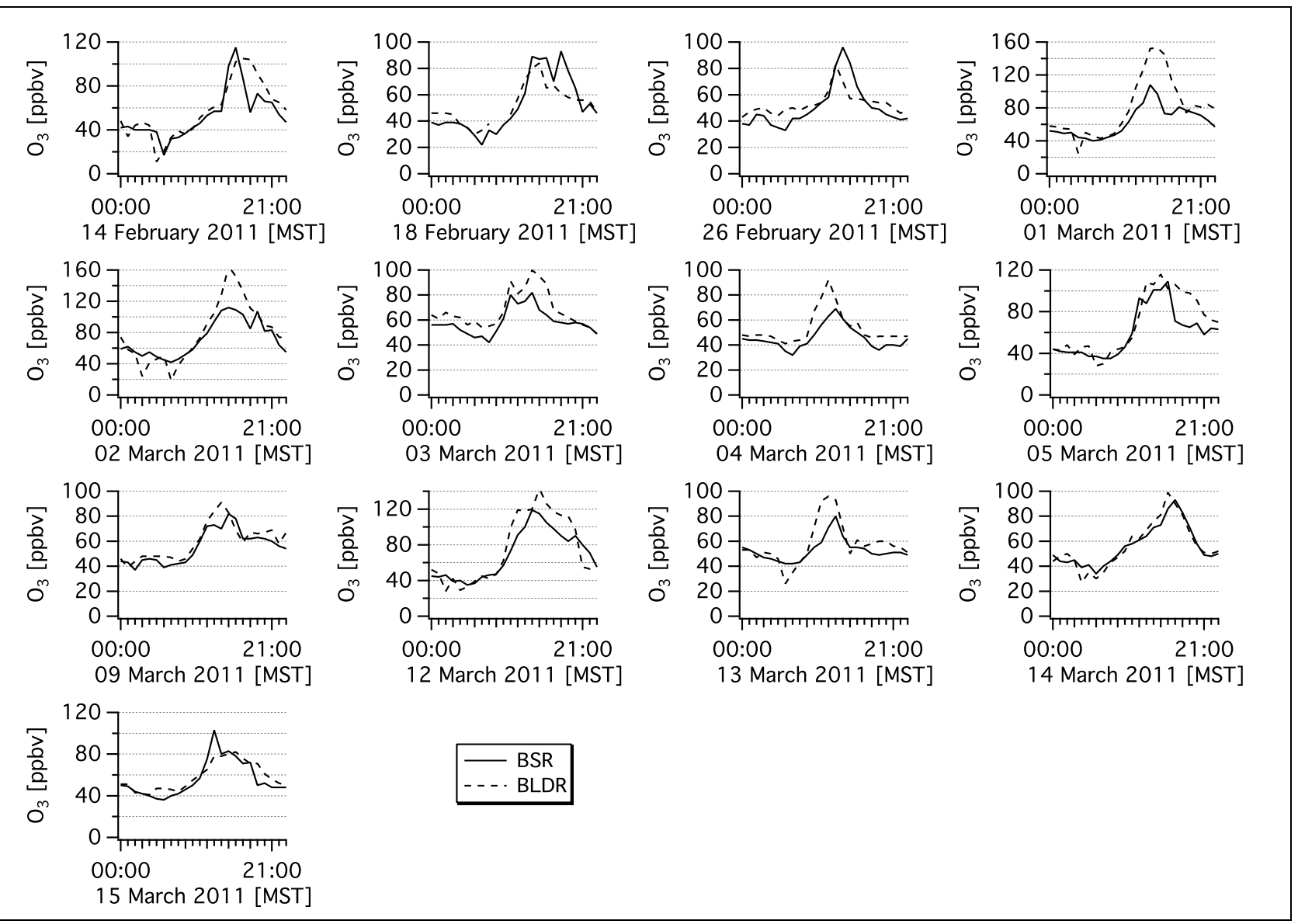

Figure $\mathrm{S}_{5} \mathrm{O}_{3}$ mixing ratios during 13 episodes at Boulder sites February to March 2011 


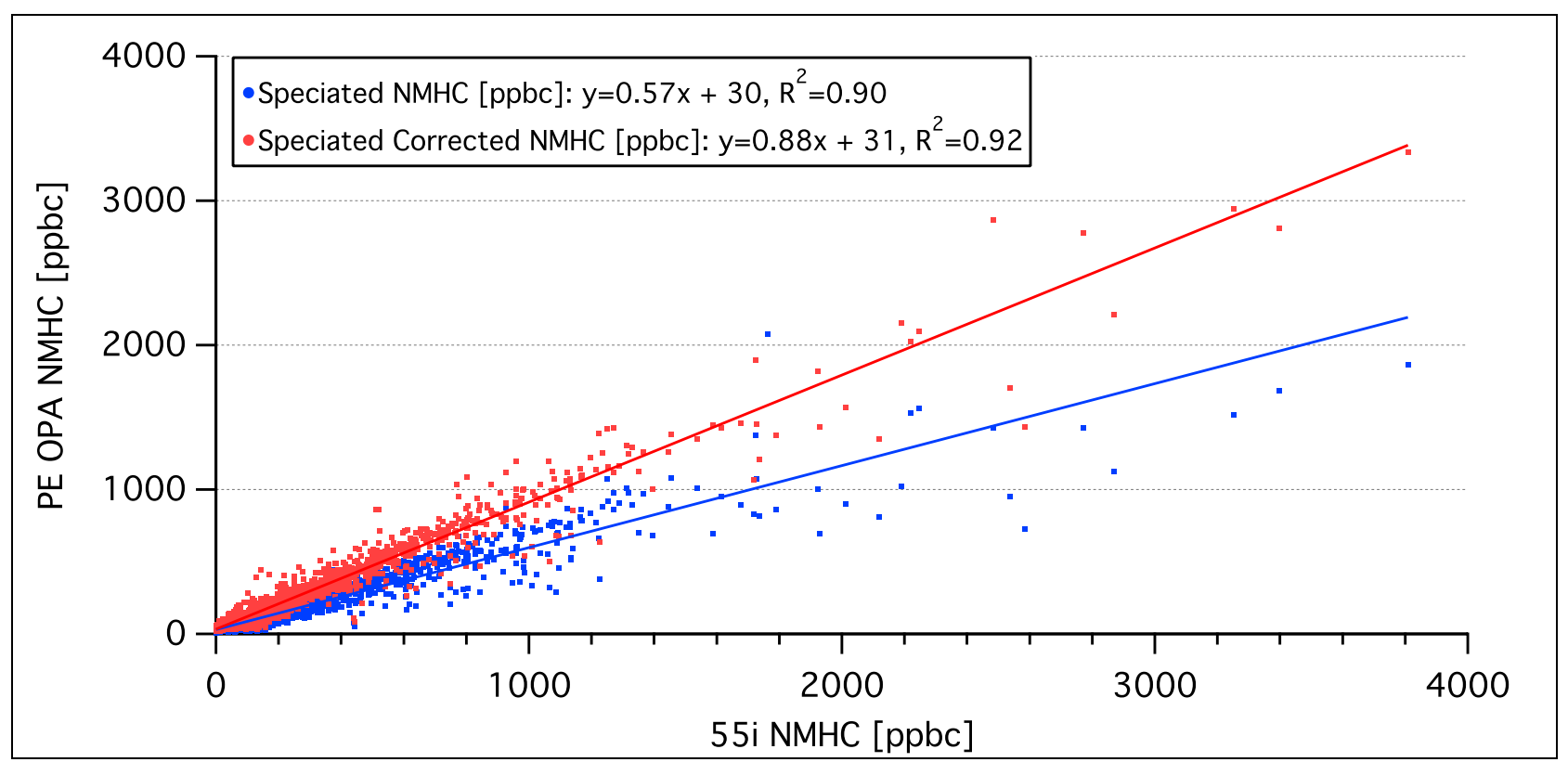

Figure S6 Sum of speciated NMHC as ppbC versus total NMHC as ppbC during the period October 2010 to March 2011 at 2012 Boulder South Road (BSR) 


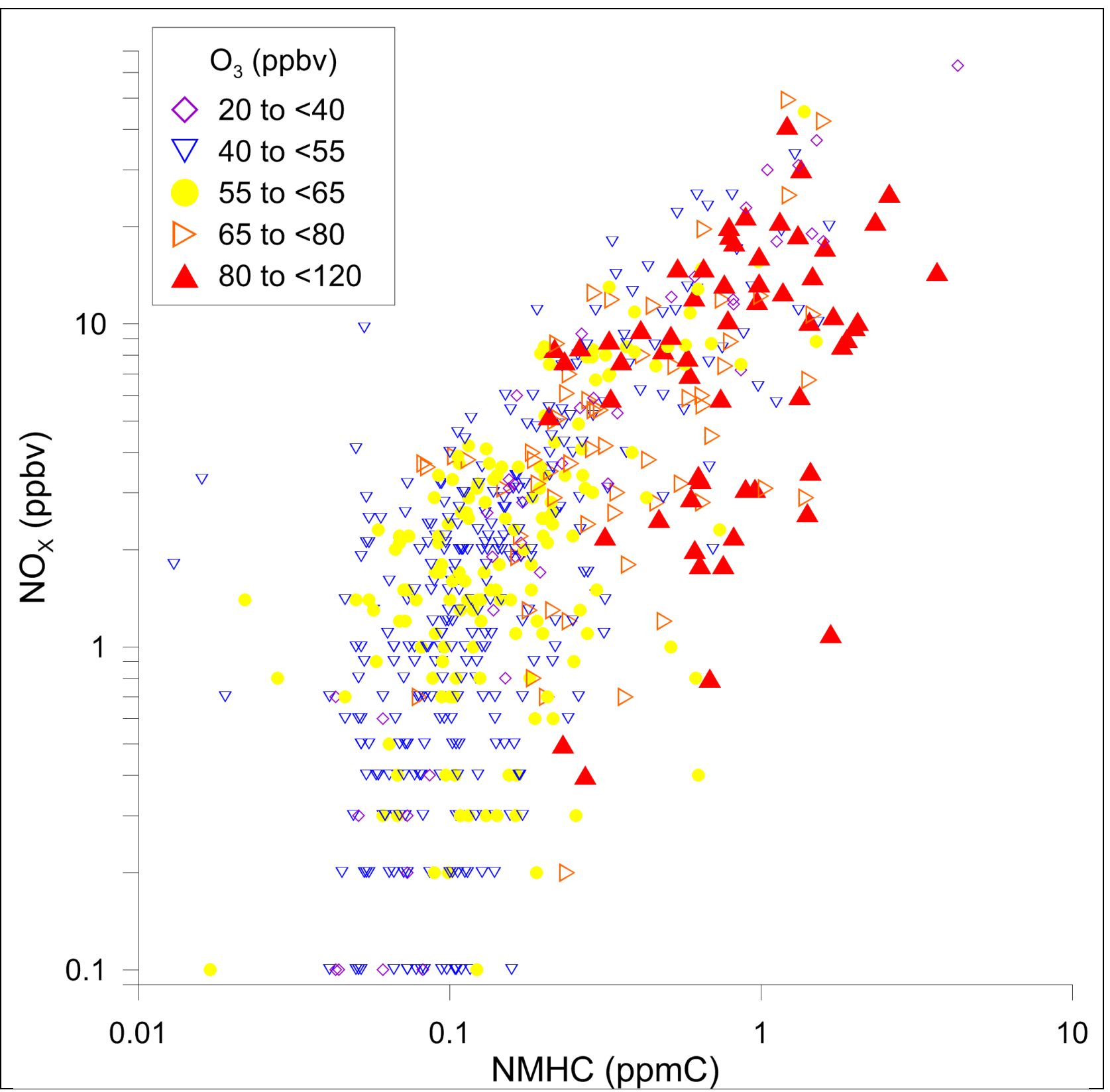

Figure $\mathrm{S}_{7} \mathrm{O}_{3}, \mathrm{NMHC}$ and $\mathrm{NO}_{\mathrm{x}}$ during daytime for the period January to March 2011 at Wyoming DEQ Boulder site (BLDR) 

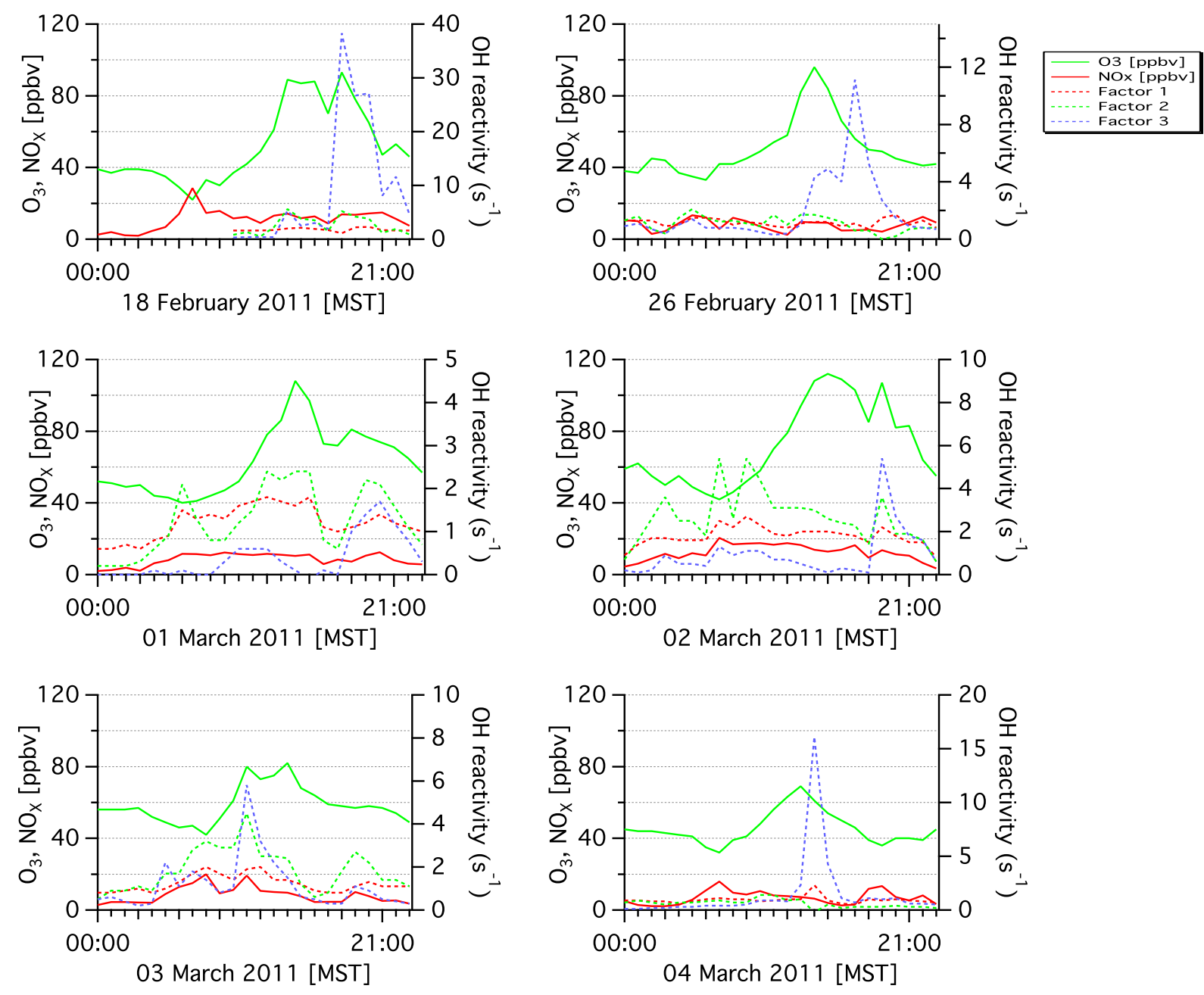

Figure S8 Relationship of PMF factor $\mathrm{OH}$ reactivity, $\mathrm{NO}_{x}$ and $\mathrm{O}_{3}$ during selected episodes at Boulder South Road (BSR) 

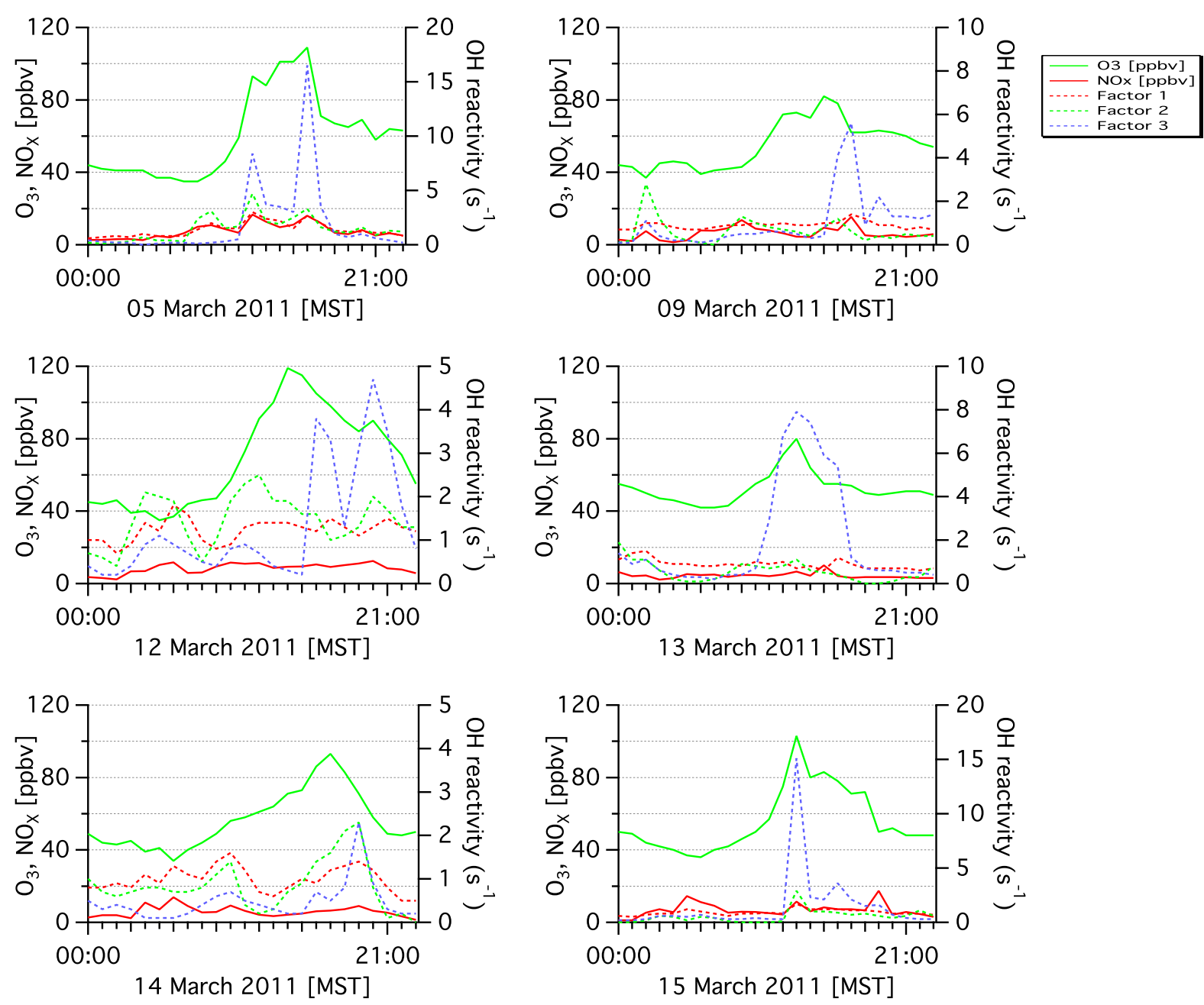

Figure S8 (continued) Relationship of PMF factor $\mathrm{OH}$ reactivity, $\mathrm{NO}_{\mathrm{x}}$ and $\mathrm{O}_{3}$ during selected episodes at Boulder South Road (BSR) 
Table S9 Mixing ratios, $\mathrm{OH}$ reaction rate coefficients at $298.15 \mathrm{~K}$ and $1013.25 \mathrm{hPa}$, and $\mathrm{OH}$ reactivity for two selected hours at the Boulder South Road (BSR)

\begin{tabular}{|c|c|c|c|c|c|c|}
\hline & $\begin{array}{l}2 / 20 / 2011 \\
\text { at 18:00 } \\
\text { (ppbv) }\end{array}$ & $\begin{array}{l}2 / 18 / 2011 \\
\text { at 18:00 } \\
\text { (ppbv) }\end{array}$ & Ref. & $10^{12} \mathrm{k}_{\mathrm{OH}}$ & $\begin{array}{l}2 / 20 / 2011 \text { at } \\
18: 00 \\
\text { OH reactivity } \\
\left(\mathrm{s}^{-1}\right)\end{array}$ & $\begin{array}{l}2 / 18 / 2011 \text { at } \\
18: 00 \\
\text { OH reactivity } \\
\left(\mathrm{s}^{-1}\right)\end{array}$ \\
\hline $\mathrm{O}_{3}$ & 35 & 93 & & & & \\
\hline $\mathrm{CO}$ & 169 & 297 & 5 & 0.2 & 0.83 & 1.46 \\
\hline $\mathrm{CH}_{4}$ & 1930 & 3730 & 1 & 0.0064 & 0.30 & 0.59 \\
\hline ethane & 1.97 & 99.97 & 1 & 0.25 & 0.01 & 0.62 \\
\hline ethene & 0.19 & 2.39 & 1 & 8.52 & 0.04 & 0.50 \\
\hline propane & 0.86 & 35.95 & 1 & 1.1 & 0.02 & 0.97 \\
\hline propene & 0.11 & 0.27 & 1 & 26.3 & 0.07 & 0.17 \\
\hline$i$-butane & 0.13 & 10.74 & 1 & 2.1 & 0.01 & 0.56 \\
\hline$n$-butane & 0.29 & 11.32 & 1 & 2.4 & 0.02 & 0.67 \\
\hline ethyne & 0.32 & 14.64 & 2 & 0.75 & 0.01 & 0.27 \\
\hline$t$-2-butene & 0.12 & 0.14 & 1 & 64 & 0.19 & 0.22 \\
\hline 1-butene & 0.02 & 0.05 & 1 & 56.4 & 0.03 & 0.07 \\
\hline$c$-2-butene & 0.00 & 0.27 & 1 & 64 & 0.00 & 0.43 \\
\hline cyclopentane & 0.02 & 0.94 & 1 & 5 & 0.00 & 0.12 \\
\hline$i$-pentane & 0.10 & 8.17 & 1 & 3.6 & 0.01 & 0.72 \\
\hline$n$-pentane & 0.05 & 7.02 & 1 & 3.8 & 0.00 & 0.66 \\
\hline 1,3-butadiene & 0.01 & 0.02 & 1 & 66.6 & 0.02 & 0.03 \\
\hline propyne & 0.01 & 0.04 & 3 & 6 & 0.00 & 0.01 \\
\hline 1-pentene & 0.02 & 0.04 & 1 & 31.4 & 0.02 & 0.03 \\
\hline 2-methylpentane & 0.01 & 5.14 & 1 & 5.2 & 0.00 & 0.66 \\
\hline 3-methylpentane & 0.00 & 3.68 & 1 & 5.2 & 0.00 & 0.47 \\
\hline$n$-hexane & 0.02 & 9.64 & 1 & 5.2 & 0.00 & 1.23 \\
\hline isoprene & 0.00 & 0.00 & 1 & 100 & 0.00 & 0.00 \\
\hline benzene & 0.15 & 11.37 & 1 & 1.2 & 0.00 & 0.34 \\
\hline cyclohexane & 0.03 & 11.93 & 1 & 7 & 0.01 & 2.06 \\
\hline$i$-octane & 0.08 & 3.05 & 1 & $3.6^{* *}$ & 0.01 & 0.27 \\
\hline$n$-heptane & 0.06 & 15.86 & 1 & 6.8 & 0.01 & 2.65 \\
\hline toluene & 0.92 & 46.26 & 1 & 3.7 & 0.08 & 4.18 \\
\hline$n$-octane & 0.02 & 17.27 & 1 & 8.1 & 0.00 & 3.44 \\
\hline ethylbenzene & 0.04 & 4.26 & 1 & 7 & 0.01 & 0.73 \\
\hline$m+p$-xylene & 0.14 & 37.86 & 1 & $19 * *$ & 0.07 & 17.71 \\
\hline styrene & 0.07 & 1.53 & 4 & 58 & 0.10 & 2.18 \\
\hline$o$-xylene & 0.05 & 7.06 & 1 & 13.6 & 0.02 & 2.36 \\
\hline nonane & 0.03 & 13.36 & 1 & 9.2 & 0.01 & 3.04 \\
\hline & & & & Total & 1.89 & 49.42 \\
\hline
\end{tabular}

